\title{
ARTICLE Remote allergen exposure elicits eosinophil infiltration into allergen nonexposed mucosal organs and primes for allergic inflammation
}

\author{
Courtney L. Olbrich ${ }^{1,2}$, Maytal Bivas-Benita ${ }^{3}$, Jason J. Xenakis ${ }^{3}$, Samuel Maldonado ${ }^{3}$, Evangeline Cornwell ${ }^{1,4}$, Julia Fink ${ }^{1,4}$, \\ Qitong Yuan ${ }^{1,4}$, Nathan Gill ${ }^{4}$, Ryan Mansfield ${ }^{1}$, Karen Dockstader ${ }^{1}$ and Lisa A. Spencer (iD) ${ }^{1,2,3}$
}

The natural history of allergic diseases suggests bidirectional and progressive relationships between allergic disorders of the skin, lung, and gut indicative of mucosal organ crosstalk. However, impacts of local allergic inflammation on the cellular landscape of remote mucosal organs along the skin:lung:gut axis are not yet known. Eosinophils are tissue-dwelling innate immune leukocytes associated with allergic diseases. Emerging data suggest heterogeneous phenotypes of tissue-dwelling eosinophils contribute to multifaceted roles that favor homeostasis or disease. This study investigated the impact of acute local allergen exposure on the frequency and phenotype of tissue eosinophils within remote mucosal organs. Our findings demonstrate allergen challenge to skin, lung, or gut elicited not only local eosinophilic inflammation, but also increased the number and frequency of eosinophils within remote, allergen nonexposed lung, and intestine. Remote allergen-elicited lung eosinophils exhibited an inflammatory phenotype and their presence associated with enhanced susceptibility to airway inflammation induced upon subsequent inhalation of a different allergen. These data demonstrate, for the first time, a direct effect of acute allergic inflammation on the phenotype and frequency of tissue eosinophils within antigen nonexposed remote mucosal tissues associated with remote organ priming for allergic inflammation.

Mucosal Immunology (2020) 13:777-787; https://doi.org/10.1038/s41385-020-0310-x

\section{INTRODUCTION}

Allergic diseases encompass a range of chronic, relapsing inflammatory disorders (e.g., atopic dermatitis, hay fever, asthma, food allergy) characterized by Th2 immunity. Their global prevalence has risen dramatically within the past several decades such that allergic diseases now affect $\sim 20 \%$ of the worldwide population and represent the most common chronic conditions in childhood. ${ }^{1}$ Frequently encountered as sequential or comorbidities, the natural history of allergic diseases, as revealed through cross-sectional and longitudinal clinical studies and animal models, suggests progressive and causal relationships between allergic disorders of the skin, lung, and gut collectively referred to as the "allergic march," and often reflective of sensitization to multiple allergens. ${ }^{2-10}$ Despite strong epidemiologic evidence for causal and bidirectional relationships between cutaneous, respiratory, and gastrointestinal allergic reactions, cellular mechanisms that link local allergic reactions to remote organs along the skin: lung:gut axis and prime for the development of subsequent allergic reactions in response to new allergens remain unknown.

Eosinophils have long been implicated in allergic diseases, and increased numbers of eosinophils have been observed within both the airways and intestinal tracts of patients with asthma or allergic rhinitis and within both skin and colon of patients with atopic eczema (reviewed in ref. ${ }^{11}$ ). Data from our lab and others' have identified phenotypically distinct populations of tissueresident eosinophils, particularly within the intestinal tract ${ }^{12,13}$ and lung, ${ }^{14,15}$ exhibiting putatively homeostatic or inflammatory phenotypes. We hypothesized that the frequency and/or functional phenotype of mucosal tissue eosinophils might be modulated in response to allergic inflammatory reactions occurring at remote organs, and that these cellular alterations might prime mucosal organs for allergic inflammation. To test this hypothesis, we modified acute models of allergic inflammation localized to the skin, airway or Gl tract to model the allergic march in mice and investigated the frequency and phenotype of tissueresident eosinophils within remote, allergen nonexposed organs along the skin:lung:gut axis. Our findings demonstrate that in allergen-sensitized mice direct challenge of the skin, lung, or gut with allergen elicited not only a local eosinophilic inflammation at the site of allergen exposure, but also increased the number and frequency of eosinophils within allergen nonexposed lung and intestine. Remote allergen-recruited airway and intestinal eosinophils exhibited an activated phenotype. Moreover, the accumulation of inflammatory eosinophils within the allergen nonexposed lung was associated with mucous secretion and an exacerbated allergic airways response to subsequent inhalation of an antigenically distinct allergen, house dust mite (HDM). These data add to our understanding of the phenotypic diversity of mucosal

\footnotetext{
${ }^{1}$ Department of Pediatrics, University of Colorado School of Medicine, Aurora, CO, USA; ${ }^{2}$ Gastrointestinal Eosinophilic Diseases Program (GEDP) and Digestive Health Institute (DHI), Colorado Children's Hospital, Aurora, CO, USA; ${ }^{3}$ Division of Allergy and Inflammation, Department of Medicine, Beth Israel Deaconess Medical Center, Boston, MA, USA and ${ }^{4}$ Department of Biology, Gordon College, Wenham, MA, USA

Correspondence: Lisa A. Spencer (Lisa.Spencer@cuanschutz.edu)
}

Received: 10 October 2019 Revised: 20 May 2020 Accepted: 20 May 2020

Published online: 9 June 2020 
tissue-resident eosinophils and reveal, for the first time, a direct relationship between local allergic inflammation and eosinophil frequency and phenotypes within remote tissues along the skin: lung:gut axis that prime for allergic inflammation. As such, these findings shed light on how local allergic manifestations of the skin, respiratory tract, or gut may predispose individuals for concurrent and sequential development of allergenically distinct allergic diseases at remote loci. Our results are therefore relevant to atopic patients at risk for progressing along the "allergic march."

\section{RESULTS}

Developing a mouse model to investigate the atopic march To investigate effects of local allergic inflammation on the number and phenotype of tissue-resident eosinophils at remote sites along the skin:lung:gut axis, we modified mouse models of local skin (atopic dermatitis), lung (allergic airways disease), and gut (food allergy) allergen exposure to achieve three acute models that elicited local eosinophilic inflammation in parallel with similar levels of peripheral blood eosinophilia (Fig. 1a). Strong systemic Th2 immunity is the classic characteristic of patients at greatest risk of progressing along the atopic march. Therefore, as a foundation for each model we utilized a standard methodology of robust systemic Th2 sensitization in mice through intraperitoneal sensitization with the soluble protein antigen ovalbumin (OVA) admixed with alum adjuvant. Among other functions, alum has been shown to elicit release of IL-33 ${ }^{16}$ that could directly influence multiple aspects of the allergic march, including eosinophil hematopoiesis and recruitment. ${ }^{17}$ Therefore, to avoid any potential confounding effects of alum, control mice throughout the study received sham sensitizations with PBS admixed with alum. Importantly, OVA-alum sensitization alone had no significant impact on eosinophil frequencies in bone marrow, spleen, or intestinal tissues (Supplementary Fig. 1).

Following systemic sensitization to OVA-alum mice were challenged with OVA on 3 consecutive days by direct administration to either the skin, lung, or gut, and tissue eosinophils within lung and gut compartments assessed 4 days after the final allergen challenge (Fig. 1a). Sham control mice were sensitized with PBS-alum followed by challenge with PBS vehicle control. For skin challenge, OVA was applied to tape-stripped skin to mimic scratching of AD patients (see "Materials and methods"). To deliver OVA directly to lower airways while avoiding direct allergen exposure to the gastrointestinal tract inherent in the standard methods of airway allergen exposures (i.e., aerosolization or intranasal instillation) mice received challenge doses of OVA aerosolized directly into the right lung using a microsprayer (see "Materials and methods"). Of note, although the endotracheal spray method avoids direct allergen exposure to the gastrointestinal tract, it is plausible that self-clearing mechanisms associated with the respiratory mucociliary escalator may indirectly deliver a fraction of the allergen to intestinal tissues, as would also occur in humans. Delivery of OVA to the gut was achieved by oral gavage.

For each of the three acute allergic disease models, tissue recovered from the respective sites of allergen exposure (i.e., skin, lung, or gut) 4 days after final allergen challenge revealed local eosinophilic infiltration. For the AD model, this was manifest as an increase in dermis eosinophils (Fig. 1b). In the allergic airway inflammation model, OVA challenge elicited cellular infiltration surrounding blood vessels and airways (Fig. 1C), an increase in total bronchoalveolar lavage (BAL) cells (not shown), and an increase in the frequency of BAL eosinophils (Fig. 1d). Likewise, OVA administration to the gut elicited an increase in jejunum eosinophils (Fig. 1e). Of note, these data are significant in themselves, as to our knowledge, this is the first report of an acute model eliciting a local intestinal eosinophilic response.
Increased numbers of jejunal eosinophils were observed both around crypts and within villi, although numbers of villus eosinophils rose more steeply (Supplementary Fig. 2a). In addition to local eosinophilic inflammation, all three models elicited a similar level of peripheral eosinophilia with eosinophils accounting for $\sim 7-10 \%$ of circulating leukocytes, compared with baseline levels of $1-3 \%$ (Fig. 1f-h).

Epicutaneous or airway allergen exposure increases the number and frequency of eosinophils within allergen nonexposed intestine of allergic mice

To explore a potential link between acute local skin and lung allergic inflammation on tissue eosinophils within remote intestinal tissues (i.e., skin:gut and lung:gut axes), eosinophils were quantified from tissue sections recovered from the center of the jejunum 4 days after the final allergen challenge. As shown in Fig. 2a, allergen challenge of the skin elicited a near doubling of the total number of eosinophils within allergen nonexposed jejunum. Increased numbers of lamina propria (LP) eosinophils were observed both in areas surrounding crypts and along the length of the villi, and increased from an average of $2.16 \pm 0.89$ eosinophils per crypt:villus unit (cvu) to $3.65 \pm 0.90$ eosinophils per $\mathrm{cvu}$ in sham control versus remote allergen-challenged mice, respectively. Likewise, allergen exposure to the lower airways more than doubled the total number of eosinophils within allergen nonexposed jejunum $(2.36 \pm 0.79$ eosinophils per $\mathrm{cvu}$ in sham control versus $6.51 \pm 2.54$ eosinophils per cvu in remote allergen-challenged mice, Fig. 2b). To confirm jejunum tissues are broadly representative of small intestinal tissue eosinophil counts, in some experiments eosinophils were also quantified from ileal tissues (Supplementary Fig. 3). Of note, despite similar levels of peripheral eosinophilia (see Fig. 1f-h), allergen exposure to the lung elicited stronger eosinophilic accumulation within the gut than allergen exposure to the skin (1.7-fold versus 2.8-fold increases elicited by remote skin and airway allergen exposures, respectively). Similar to the pattern of eosinophilic infiltration observed following direct oral gavage of OVA, remote allergenelicited intestinal eosinophil infiltration favored eosinophil infiltration of villi (Supplementary Fig. 2b, c). Analyses of esophageal tissues at this time point did not reveal a substantial increase in eosinophils in either challenge model (not shown).

Using standard methodologies for isolation of intestinal leukocytes, we previously demonstrated that eosinophils are recovered from both intraepithelial (IE) and LP compartments. ${ }^{12}$ To further assess compartmentalization of eosinophils infiltrating intestinal tissues in response to remote (i.e., lung or skin) allergen challenge, LP and IE leukocytes were isolated from whole small intestine preparations 4 days after the final airway allergen challenge and analyzed by flow cytometry. In support of the histological eosinophil counts, remote allergen challenge increased the frequencies of both IE (Fig. 2C, upper) and LP (Fig. 2c, lower) eosinophils. In contrast, percentages of $\mathrm{CD}^{+}$, $\mathrm{CD}^{+}$, and $\mathrm{CD}_{11 \mathrm{c}^{+} \text {SiglecF }}{ }^{-}$(dendritic) cells in both $\mathrm{LP}$ and $\mathrm{IE}$ compartments remained static following OVA challenge (Fig. 2d-f).

Remote allergen-elicited intestinal eosinophils take on the phenotype of resident intestinal tissue eosinophils There is an increasing recognition that tissue-resident eosinophils exist in phenotypically distinct subsets that may shed light on their diverse functional roles. ${ }^{12,14,15}$ We previously demonstrated that at baseline intestinal LP and IE eosinophil populations are phenotypically distinguishable, both from blood eosinophils and from each other, based on expression levels of surface markers including the adhesion molecules CD11b and CD11c. ${ }^{12}$ Analyses of small intestinal leukocytes from sham control and OVAchallenged mice revealed the expected phenotypes demarcating 
$\mathbf{a}_{\text {Day }}$

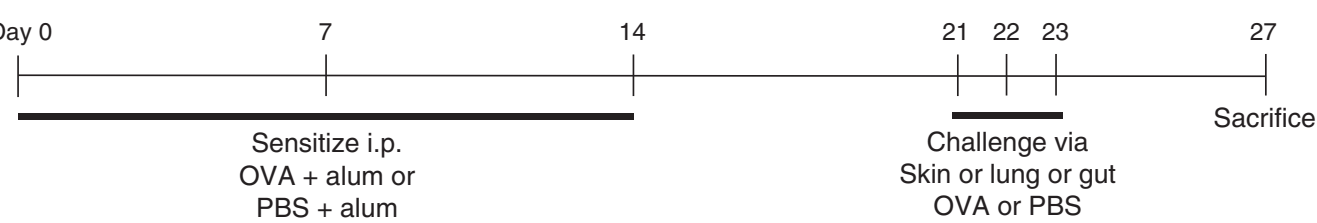

b
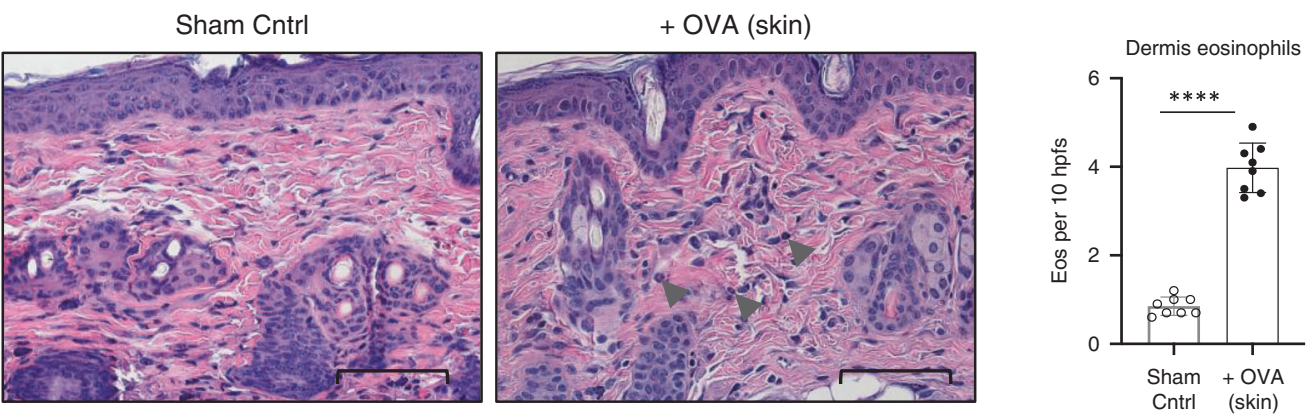

C

Sham Cntrl

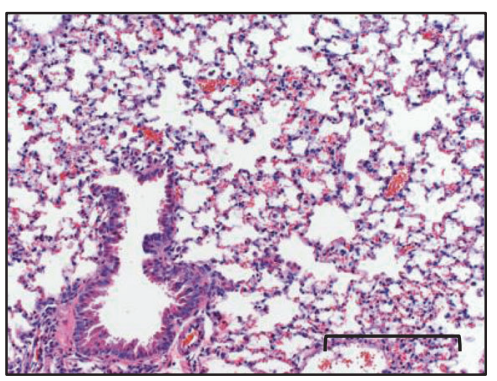

e

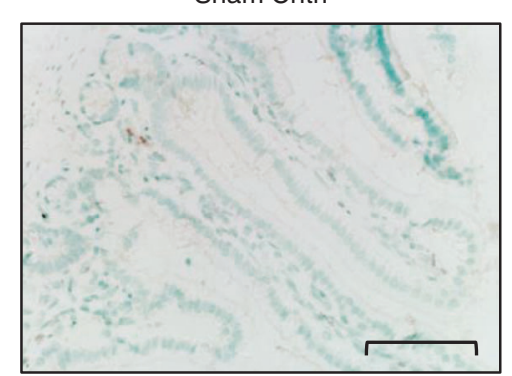

f



g
+ OVA (lung)

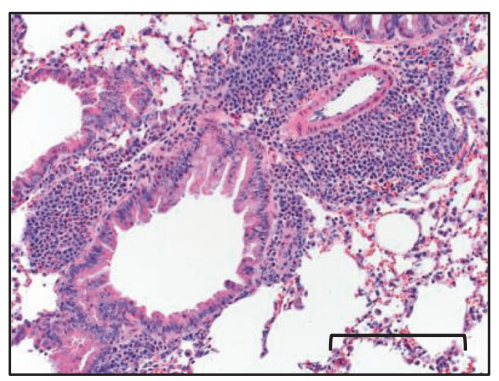

+ OVA (gut)
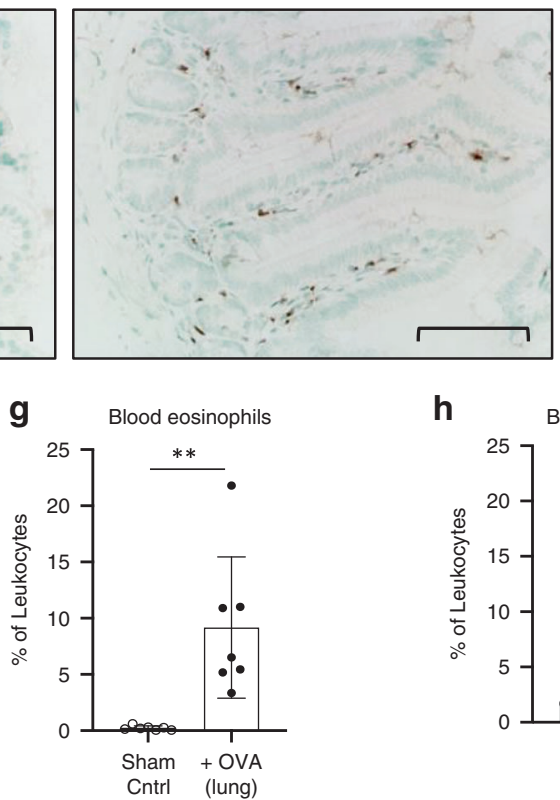

d

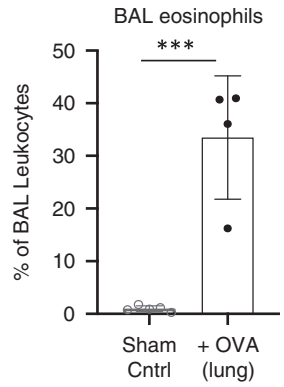

Jejunum eosinophils

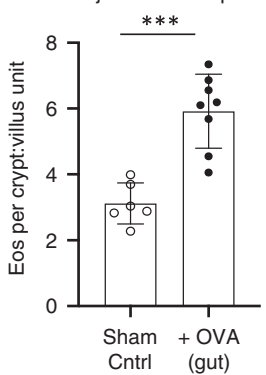

h



Fig. 1 Mouse models of acute local and systemic eosinophilic infiltration. a Experimental timeline. Mice were sensitized with three intraperitoneal injections of OVA-alum followed 1 week later by three consecutive challenges through epicutaneous, lower airways, or oral routes with OVA. Sham control mice were sensitized with PBS-alum followed by challenges with PBS. Mice were sacrificed 4 days after the final allergen challenge and eosinophils quantified from the site of allergen challenge (b-e) or peripheral blood (f-h). $\mathbf{b}$ Eosinophils were quantified from H\&E-stained sections of the dermis following allergen administration to tape-stripped skin. Following allergen administration to the lower airways lung tissue sections revealed cellular infiltrates (c) and induction of BAL fluid eosinophilia, as quantified by flow cytometry (d). e Oral gavage of allergen elicited increased numbers of eosinophils within intestinal jejunum, as quantified from $\alpha \mathrm{MBP}$ immunohistochemistry. Allergen challenge to the skin (f), lung (g), or gut (h) also elicited an increase in peripheral eosinophils. Data shown are from one representative experiment (d) or combined data from two independent experiments (b, e, f-h). b, e Magnification $\times 400$, scale bar $80 \mu \mathrm{m}$. c $\times 200$, scale bar $200 \mu \mathrm{m}$. Error bars show standard deviation. ${ }^{* *} p<0.001 ;{ }^{* *} p<0.0001$. 

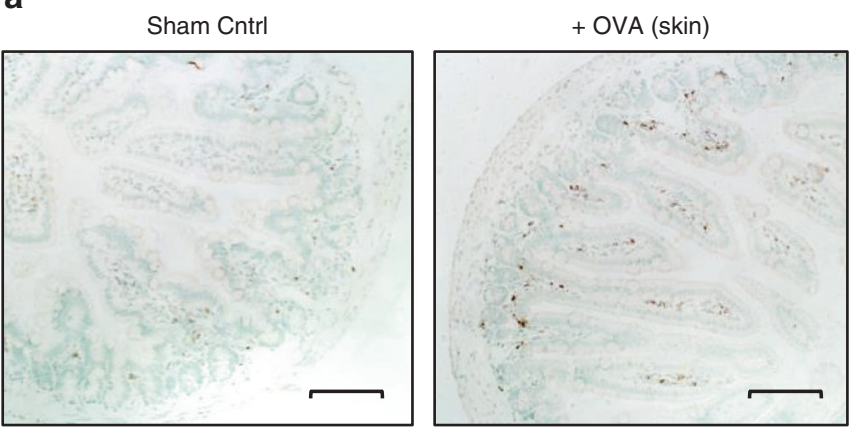

Jejunum eosinophils post skin challenge

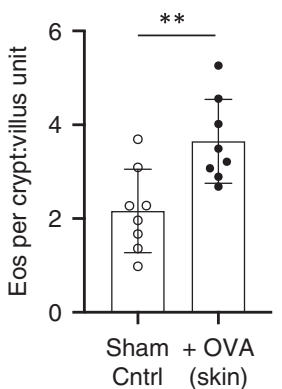

Jejunum eosinophils post lung challenge

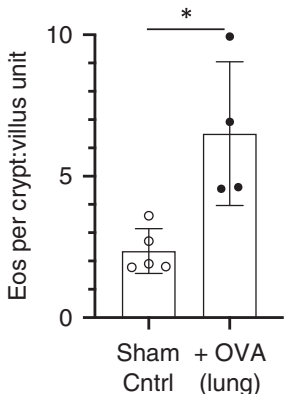

C



d


e


f
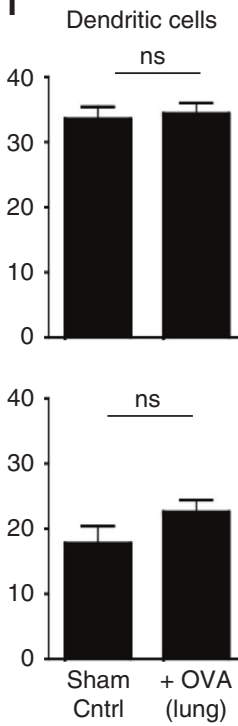

g

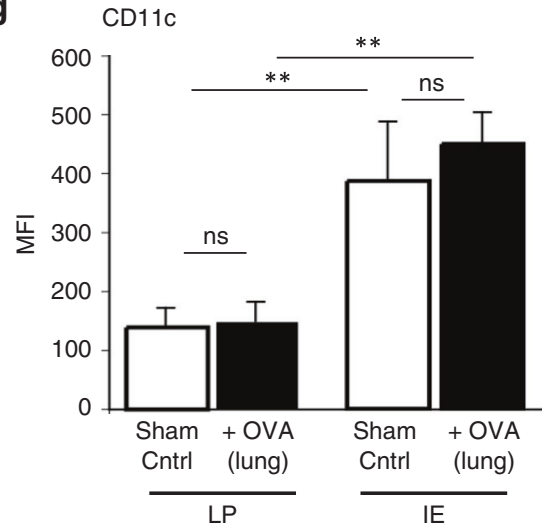

h



Fig. 2 Epicutaneous or endotracheal allergen challenge elicits an increase in eosinophils within remote, allergen nonexposed intestine. Eosinophils were quantified from anti-MBP-stained jejunum tissue sections recovered 4 days after the final allergen challenge to tape-stripped skin (a) or lower airways (b). Frequencies of eosinophils (c), CD4 ${ }^{+} \mathrm{T}$ cells (d), CD8 ${ }^{+} \mathrm{T}$ cells $(\mathbf{e})$, and dendritic cells (f) were further quantified by flow cytometry of single-cell suspensions of intraepithelial (IE, upper panels) or lamina propria (LP, lower panels) compartments recovered from whole small intestine using the following gating strategies: eosinophils, live, $\mathrm{CD} 45^{+} \mathrm{SSC}^{\text {hi }}$ SiglecF ${ }^{\text {hi }}$. CD4 ${ }^{+} \mathrm{T}_{\mathrm{cells}}$, live, $\mathrm{CD} 45^{+} \mathrm{CD} 3^{+} \mathrm{CD} 4^{+}$ cells; $\mathrm{CD} 8^{+} \mathrm{T}$ cells, live, $\mathrm{CD}_{4} 5^{+} \mathrm{CD} 3^{+} \mathrm{CD} 8^{+}$cells; and dendritic cells, live, $\mathrm{CD} 45^{+}$SiglecF $\mathrm{CD}^{-} 1 \mathrm{c}^{+}$cells and expressed as percentage of $\mathrm{CD} 45^{+}$ leukocytes. g Gated eosinophils from IE and LP preparations were further assessed for their expression of surface CD11C (left panel) and CD11b (right panel) that distinguish resident IE and LP eosinophils. Data are expressed as average change in mean fluorescence intensity (MFI) of relevant over isotype control antibodies. c-f $N \geq 4$. Sham control mice were sensitized with PBS-alum and challenged with PBS. In a magnification $\times 200$; scale bars, $100 \mu \mathrm{m}$. Error bars show standard deviation. ${ }^{*} p<0.01 ;{ }^{* *} p<0.001$.

IE and LP eosinophils and demonstrated that remote allergenelicited eosinophils had acquired tissue-specific surface markers appropriate to their respective localizations within intestinal tissues (Fig. 2g, h).
Both epicutaneous and oral allergen exposure elicit eosinophil infiltration into the lung

It is now recognized that low levels of eosinophils home to and reside within the healthy lung at steady state. ${ }^{14}$ Therefore, we 
queried whether remote exposure of the skin or gut might impact basal numbers of lung tissue eosinophils (i.e., skin:lung and gut: lung axes). Similar to the effect of remote (i.e., skin or lung) allergen exposure on intestinal tissue eosinophils, allergen challenge of either the skin or gut elicited an increase in the number of eosinophils within lung tissue 4 days after final allergen challenge (Fig. 3a, b). In contrast to the sparse distribution of eosinophils throughout the lung parenchyma at baseline (Fig. 3a, $b$ left panels), remote allergen-elicited lung eosinophils could be observed localized around venules (Fig. 3a, b right upper panels), near airspaces (Fig. 3a right lower panel), and in small clusters within the parenchyma (Fig. 3b, right lower panel), and were not displaced by lung perfusion. Increased frequencies of eosinophils were detected within the lung as early as 1 day after allergen challenge (Fig. 3c). Similar to the more robust effect elicited by the lung:gut axis compared with the skin:gut axis in promoting intestinal eosinophils, remote allergen challenge by oral gavage elicited a stronger lung eosinophil infiltration than allergen challenge to the skin (3.5-fold versus 1.8-fold increase over baseline following oral gavage or skin challenge, respectively).

Intestinal tissue eosinophils, but not lung eosinophils, remain elevated at least 11 days post final skin allergen exposure

To begin to investigate the longevity of remote allergen-induced increases in tissue eosinophils, allergen-sensitized, and skinchallenged mice were sacrificed 11 days after the final allergen challenge and numbers of lung and jejunum tissue eosinophils quantified. As shown in Fig. 4, mice continued to exhibit statistically higher numbers of jejunum eosinophils up to 11 days post final allergen exposure to the skin while numbers of lung eosinophils within the same animals had returned to baseline by this time point.

Oral allergen-elicited lung-infiltrating eosinophils exhibit an inflammatory phenotype and are associated with airway mucus production

As described in the "Introduction", the phenotype of resident lung eosinophils resembles blood eosinophils and they are believed to serve homeostatic functions at baseline. ${ }^{14,18}$ Within the context of an allergic airways response, recruited lung eosinophils take on an inflammatory phenotype, including the induction of CD11c expression and upregulation of the sialic acid binding lectin SiglecF. ${ }^{19}$ To determine the phenotype of lung-infiltrating eosinophils elicited by remote allergen exposure PBS-perfused lungs were digested and eosinophils phenotyped by flow cytometry. Confirming our histological findings, oral allergen exposure increased the frequency of eosinophils within lung tissues (Fig. 5a, b). Control mice exhibited the expected single population of resident, homeostatic eosinophils (hEos), defined as $\mathrm{CD} 45^{+} \mathrm{SSC}^{\text {hi }}$ SiglecF ${ }^{\mathrm{lo}} \mathrm{CD} 11 \mathrm{c}^{-}$cells (Fig. 5a, left panel). In contrast, following intragastric allergen exposure two distinct populations of lung tissue eosinophils were observed, the first identical to resident homeostatic lung eosinophils of control mice, and the second exhibiting the surface marker phenotype of activated, "inflammatory" lung eosinophils (iEos), i.e., CD45 ${ }^{+} \mathrm{SSC}^{\text {hi }}$ Sigle$\mathrm{CF}^{\mathrm{int}} \mathrm{CD} 11 c^{\mathrm{int}}$ (Fig. 5a, right panel). Of note, the appearance of iEos in the remote allergen-challenged mice was not accompanied by a loss of hEos, suggesting hEos are not converting to iEos within the lung tissue (Fig. 5c).

Inflammatory eosinophils infiltrating the lungs within the context of allergic airway inflammation promote goblet cell hyperplasia and enhanced mucus production. ${ }^{20,21}$ Therefore, we queried whether oral allergen-elicited iEos might be accompanied by an increase in mucus secretion. To test this hypothesis tissue sections were stained with periodic acid-Schiff (PAS) stain to detect carbohydrates. Although none of the lung sections recovered from sham control mice $(N=16)$ exhibited PAS staining, lung sections from 12 of $16(75 \%)$ of the oral allergen- challenged mice exhibited evidence of airway mucus secretion (Fig. 5d-f). Quantification of mucus positive airways across all mice confirms a statistically significant induction of airway mucus in oral allergen challenged mice (Fig. 5f). Intriguingly, airway mucus secretion was not readily detected in lungs of mice following skin challenge (not shown).

Oral or epicutaneous allergen exposure primes the airways for a more robust allergic response to inhaled house dust mite Our findings that oral allergen challenge elicits inflammatory eosinophils within the lung associated with mucus secretion suggested that remote allergen exposure might predispose for exacerbated airways inflammatory reactions to inhaled allergens. To test this hypothesis, we utilized the acute oral allergen challenge model with the modification that 5 days after the final oral OVA challenge mice received intranasal administrations of HDM, a common allergen implicated in human asthma (Fig. 6a). Compared with sham-treated controls, OVA-sensitized mice challenged with intragastric OVA exhibited more robust allergic airways inflammation in response to subsequent intranasal administrations of HDM, as evidenced histologically by cellular infiltrations (Fig. 6b) and an overall increase in the total number of cells recovered from the BAL, comprised of a higher frequency and total number of eosinophils (Fig. 6c).

Although parallel experiments using india ink confirm dye are confined to the stomach following the oral gavage technique (Supplementary Fig. 4c), we cannot completely rule out the possibility of microaspiration in individual mice during oral gavage, thereby inadvertently delivering allergen to the airways. ${ }^{22}$ Therefore, to confirm that the remote allergen-elicited increased sensitivity to a subsequent inhaled allergen was not caused by microaspiration, and to confirm applicability to both the gut:lung axis as well as the skin:lung axis, we subjected the acute skin challenge model to subsequent HDM exposure. Similar to orally challenged mice, OVA skin-challenged mice exhibited a more robust eosinophilic airway response to inhaled HDM than shamtreated control mice (Fig. 6d).

\section{DISCUSSION}

Bidirectional, progressive relationships link allergic diseases of the skin (e.g., atopic dermatitis), respiratory tract (e.g., asthma, allergic rhinitis), and gut (e.g., food allergy, eosinophilic gastrointestinal diseases (EGIDs)). However, impacts of local allergic inflammation on the cellular landscape of remote mucosal organs along the skin:lung:gut axes are unknown. Here, we utilized acute mouse models of allergic dermatitis, allergic airway inflammation, and food allergy to investigate impacts of acute allergic reactions on the frequency and phenotype of tissue eosinophils within remote, allergen nonexposed organs. Our data demonstrate that in allergen-sensitized mice epicutaneous, airway or intragastric allergen exposure elicits not only a local eosinophilic inflammation at the site of allergen challenge, but also increases the number and frequency of activated tissue eosinophils within allergen nonexposed intestine and lung. Accumulation of activated eosinophils within the lung following remote epicutaneous or oral allergen challenge was associated with an exacerbated allergic airways response to subsequent inhaled HDM. These data demonstrate for the first time that local allergic inflammatory reactions alter the composition of tissue eosinophil compartments within remote mucosal organs, offering new insights into the phenotype and plasticity of mucosal tissue eosinophils and the crosstalk between organs along the skin:lung:gut axes that drives progression of allergic diseases.

Administration of OVA either to tape-stripped skin or directly into the lower airways of OVA-sensitized mice increased both the number and frequency of intestinal tissue eosinophils. Both acute disease models induced a concurrent increase in circulating 
a
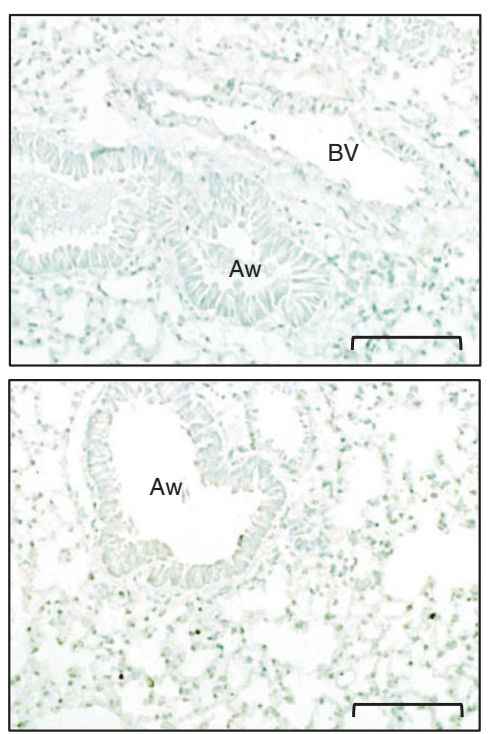

b
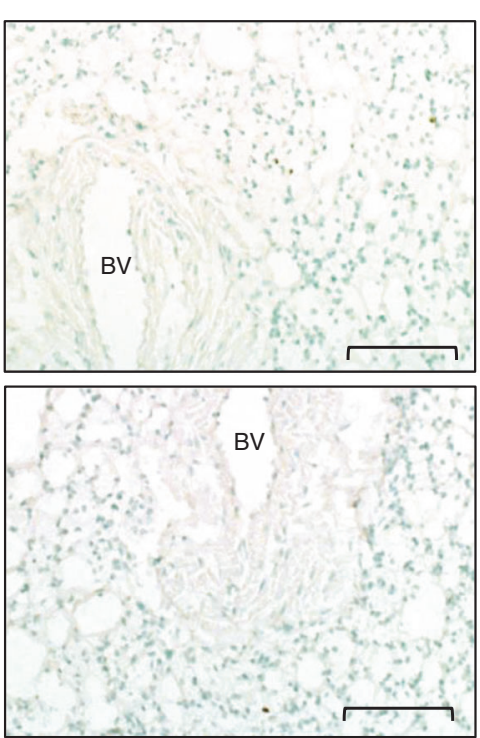

C

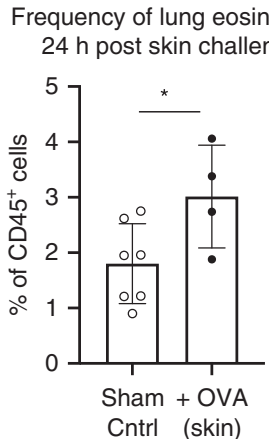

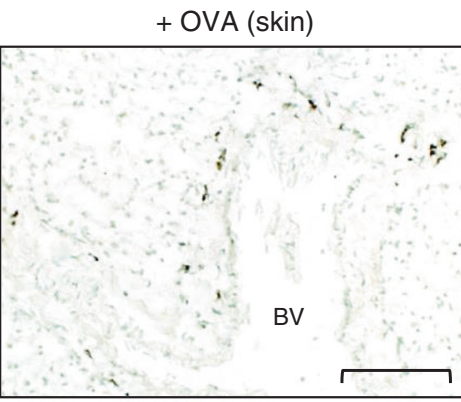
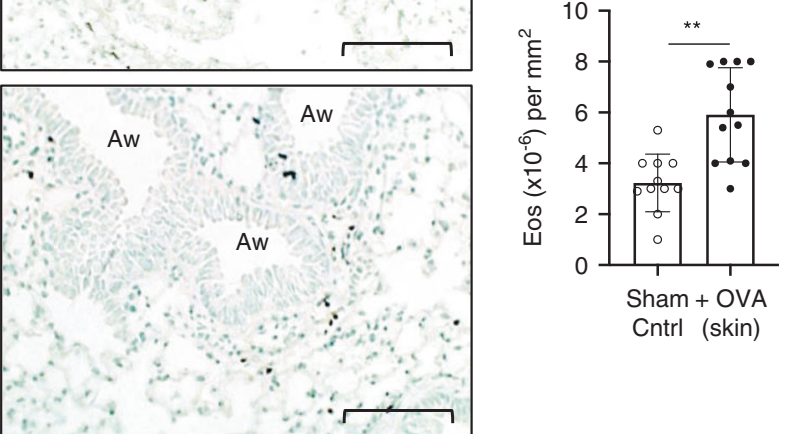

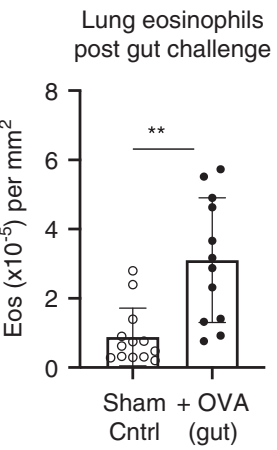

Fig. 3 Epicutaneous or intragastric allergen challenge elicits an increase in eosinophils within remote, allergen nonexposed lung. a, b Eosinophils were quantified from anti-MBP-stained nonperfused (a) or PBS-perfused (b) lung tissue sections recovered 4 days after the final allergen challenge to tape-stripped skin (a) or gut (b). Combined data from two independent experiments are shown. PBS-perfused lungs from mice $24 \mathrm{~h}$ after final allergen challenge were digested and frequency of lung eosinophils determined by flow cytometry (c). Sham control mice were sensitized with PBS-alum and challenged with PBS. a, b Magnification $\times 200$; scale bar, $100 \mu \mathrm{m}$. BV blood vessel, Aw airway. Error bars show standard deviation. ${ }^{*} p<0.05 ;{ }^{* *} p \leq 0.005$. 
a

Jejunum eosinophils

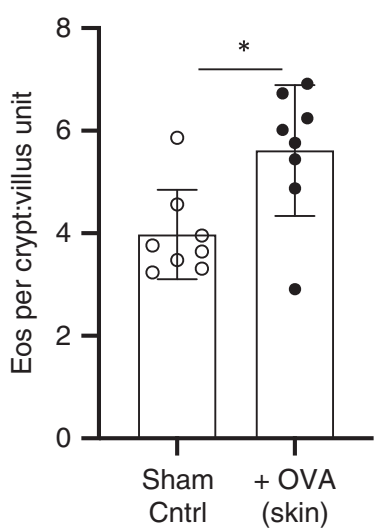

b

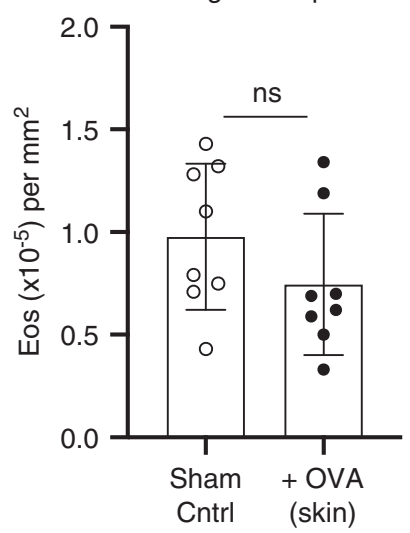

Fig. 4 Numbers intestinal eosinophils, but not lung eosinophils, remain elevated up to 11 days following skin allergen challenge. a PBS-alum (sham) or OVA-alum sensitized mice received 3 consecutive epicutaneous challenges with PBS (sham) or OVA as in Fig. 1a with the modification that all mice were sacrificed on day 34 (11 days after the final skin allergen challenge). Intestinal (a) and lung (b) eosinophils were quantified from anti-MBP stained tissue sections. ${ }^{*} p<0.01$.

eosinophils. Since eosinophils exiting the bone marrow express the gut-homing integrin $a 4 \beta 7$ and home naturally to the intestinal tract at baseline, ${ }^{23}$ it is tempting to speculate an increased level of circulating eosinophils as sufficient for remote allergen-induced intestinal eosinophil recruitment. However, levels of circulating eosinophils do not directly correlate with tissue-accumulated eosinophils since, despite similar levels of circulating eosinophils, allergen exposure to the lung elicited stronger eosinophil accumulation within the jejunum than did epicutaneous allergen exposure. Moreover, peripheral eosinophilia by itself does not always correspond with intestinal eosinophil numbers as demonstrated by Mishra et al. wherein eosinophilic airway inflammation elicited by repeated inhalation of Aspergillus fumigatus was accompanied by an increase in peripheral eosinophilia with no impact on numbers of intestinal eosinophils. ${ }^{24}$ Of note, the Mishra et al. studies were performed in mice on the $129 \mathrm{SvEv}$ background. Further studies are warranted to define mechanisms that regulate eosinophil infiltration and accumulation within remote organs, including studies that parse relative contributions of mouse strain, nature of the allergen and mode of sensitization (i.e., sensitization via mucosal exposure versus systemic sensitization). Indeed our findings are in agreement with Bui et al. who reported an increase in intestinal eosinophils following aerosolized OVA challenge of systemically sensitized BALB/C mice (although ingestion of inhaled allergen cannot be ruled out in the Bui et al. study). ${ }^{25}$ Further comprehensive studies are underway in our mouse models of the allergic march to identify systemic networks of cytokines and their tissue targets that shape resident tissue eosinophil compartments in remote organs. Recent studies suggest signaling axes involving epithelial cell-derived TSLP, IL-33, and/or IL-25 and mucosal tissue ILC2 and Tuft cells may play important, yet complex roles. ${ }^{26,27}$

Biological significance of the remote allergen-induced influx of intestinal eosinophils remains to be determined. Approximately $64 \%$ of patients with eosinophilic esophagitis (EoE), ${ }^{28}$ and $39-42 \%$ of patients with other (non-EoE) EGIDs ${ }^{29}$ (i.e., eosinophilic gastroenteritis, eosinophilic colitis) exhibit a co-existing or previous atopic disease; it is therefore plausible that remote allergen-elicited impacts on intestinal eosinophil populations might prime for the development of EGIDs. The presence of IE eosinophils is considered a negative prognostic indicator in EGIDs; therefore the predominance of remote allergen-elicited villus- migrated eosinophils in the LP (Supplementary Fig. 2) taken together with an increased frequency of IE-associated eosinophils (Fig. 2c) might further support a connection between remote allergen exposure and EGID susceptibility and/or severity. A similar scenario may also help to explain the observed increased incidence of EoE in food allergy patients receiving repetitive allergen administrations through oral immunotherapy (OIT). ${ }^{30,31}$ Of note, in our acute models of skin or lung allergen challenge we did not observe substantial numbers of eosinophils infiltrating the esophagus. It will be interesting to determine whether a chronic model of allergen exposure to the skin or lungs might also elicit esophageal eosinophils. Indeed, epicutaneous routes of repetitive allergen exposure have proven a successful mechanism of sensitization for mouse models of EoE. ${ }^{32,33}$

Here, we also show that epicutaneous or intragastric allergen exposure modulates lung tissue eosinophil populations as early as 1 day post final allergen challenge, demonstrating crosstalk along the skin:lung and gut:lung axes. Notably, there were distinct differences in the longevity of remote allergen-elicited eosinophils within airway and intestinal tissues. Analyses of intestinal and lung eosinophil populations 11 days after three consecutive skin challenges revealed a sustained increase in intestinal eosinophils, in contrast to lung eosinophil numbers in the same animals, which had returned to baseline by this time point.

At baseline resident lung eosinophils express a phenotype resembling blood eosinophils (i.e., $\mathrm{CD} 11 \mathrm{c}^{-} \mathrm{CD}_{2} \mathrm{~L}^{+}$SiglecF ${ }^{\text {lo/int }}$ ) and are believed to function in homeostatic roles. ${ }^{14,18}$ Our data shown here demonstrate that eosinophils infiltrating the lung in response to skin or gut allergen challenge exhibit an activated phenotype, including induced expression of $\mathrm{CD} 11 \mathrm{c}$ and upregulation of SiglecF, thereby donning the phenotype exhibited by inflammatory lung tissue or BAL eosinophils recovered in active allergic airways disease. ${ }^{14,19}$ Moreover, accumulation of oral allergenelicited lung eosinophils was accompanied by airway mucus production. These observations may offer mechanistic insights into clinical observations of respiratory manifestations of gastrointestinal allergies, e.g., wheezing and bronchospasm. ${ }^{34-36}$

Finally, we were interested in understanding whether acute local allergen exposure might prime remote mucosal organs for enhanced susceptibility to new allergic diseases. Therefore, we exposed epicutaneous or oral allergen-challenged mice to inhaled HDM, an allergen antigenically distinct from OVA and a significant cause of human asthma. Unlike sensitization with a soluble protein antigen such as OVA which requires an adjuvant to elicit an immune response, HDM is inherently immunogenic, presumably owing to its protease activity. Shown here, prior intragastric or epicutaneous exposure of sensitized mice to OVA primed the airways for enhanced susceptibility to HDM, as evidenced by cellular infiltrations surrounding blood vessels and airways, and BAL eosinophilia. These data may offer mechanistic insights into polysensitization and progressive allergic disease manifestations associated with patients undergoing the allergic march.

Data shown here demonstrate bidirectional relationships between local acute gastrointestinal, skin or respiratory allergic reactions and the phenotype and frequency of eosinophils within remote mucosal tissues that were further associated with organ priming for allergic inflammation in response to an antigenically distinct allergen. Collectively these findings provide new insights into the plasticity of mucosal tissue eosinophil populations and organ crosstalk relevant to allergic disease progressions.

\section{MATERIALS AND METHODS}

Animals

Studies received prior approvals from BIDMC or CUSOM Institutional Animal Care and Use Committees. Six-to-ten-week-old $\mathrm{BALB} / \mathrm{c}$ mice were from Charles River Laboratory (Wilmington, MA) and maintained under conventional specific pathogen free 
a



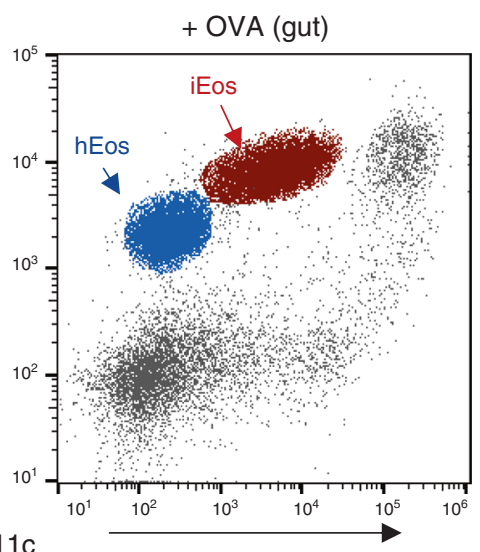

b



Cntrl (gut)

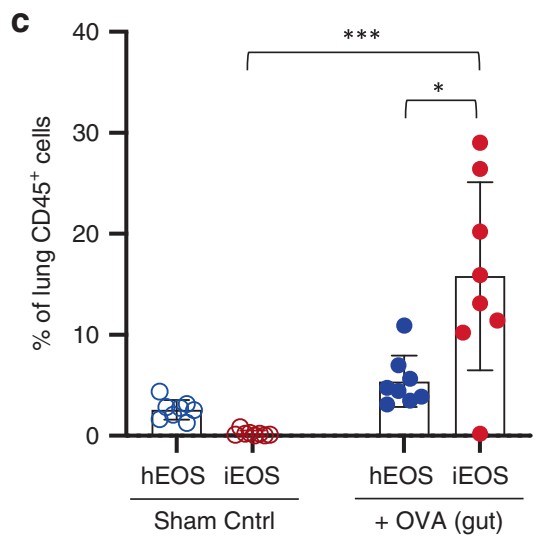

d


e + OVA (gut)


Fig. 5 Oral allergen-elicited lung eosinophils exhibit an inflammatory phenotype and their accumulation is accompanied by increased mucus production. Four days after final oral challenge, mice were sacrificed and lungs perfused with PBS. Lung tissue was digested and disaggregated and frequencies and phenotypes of lung tissue eosinophils analyzed by flow cytometry (a-c) or formalin fixed, paraffin embedded, and stained with PAS to assess mucus secretion (d-f). a-c Lung eosinophils were identified as live, $\mathrm{CD}^{4} 5^{+} \mathrm{SSC}^{\text {hi }}$ SiglecF ${ }^{+}$cells after gating out $\mathrm{CD} 11 \mathrm{c}^{\mathrm{hi}}$ alveolar macrophages. Within the gated population eosinophils were further categorized as homeostatic (hEos, SiglecF ${ }^{\mathrm{lo}} \mathrm{CD} 11 \mathrm{c}^{-}$) or inflammatory (iEos, SiglecF $\left.{ }^{\text {hi }} \mathrm{CD} 11 \mathrm{c}^{\mathrm{int}}\right)(\mathbf{a}, \mathbf{c})$. Sham control mice were sensitized with PBS-alum and challenged with PBS. Magnification $\times 100$ (d, e); $\times 200$ (inset: d, e). Scale bar, $200 \mu \mathrm{m}$. ${ }^{*} p<0.05 ;{ }^{* *} p \leq 0.005,{ }^{* * *} p \leq 0.0005$. $P$ values determined by student's $t$ test $(\mathbf{b}, \mathbf{f})$ or two-way ANOVA with Tukey's multiple comparisons test (c). 




b

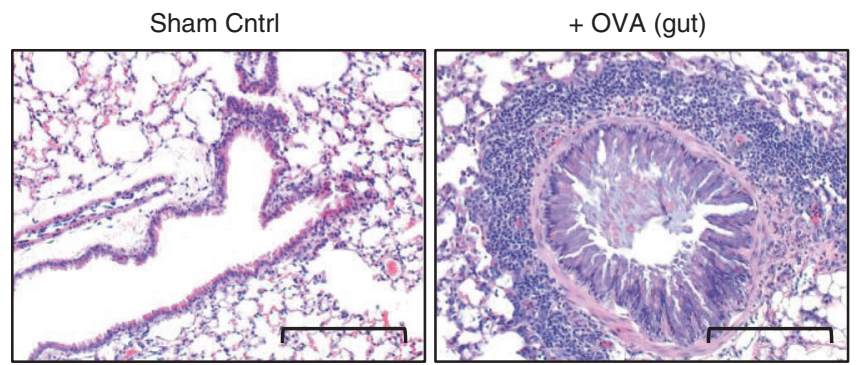

C

Total BAL cells

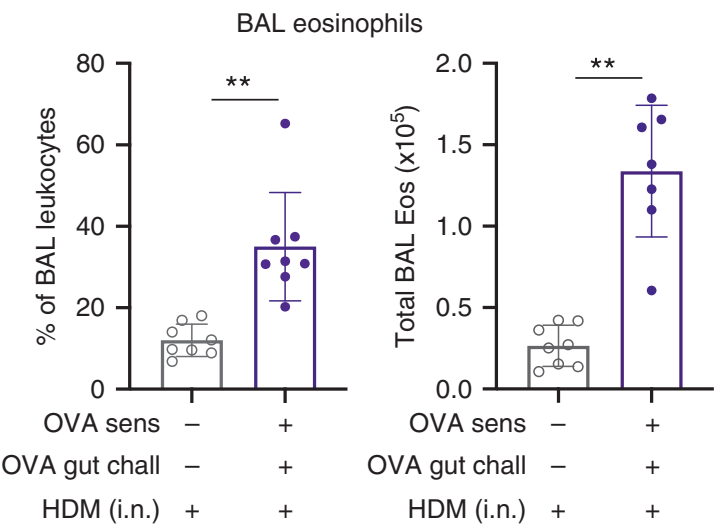

d
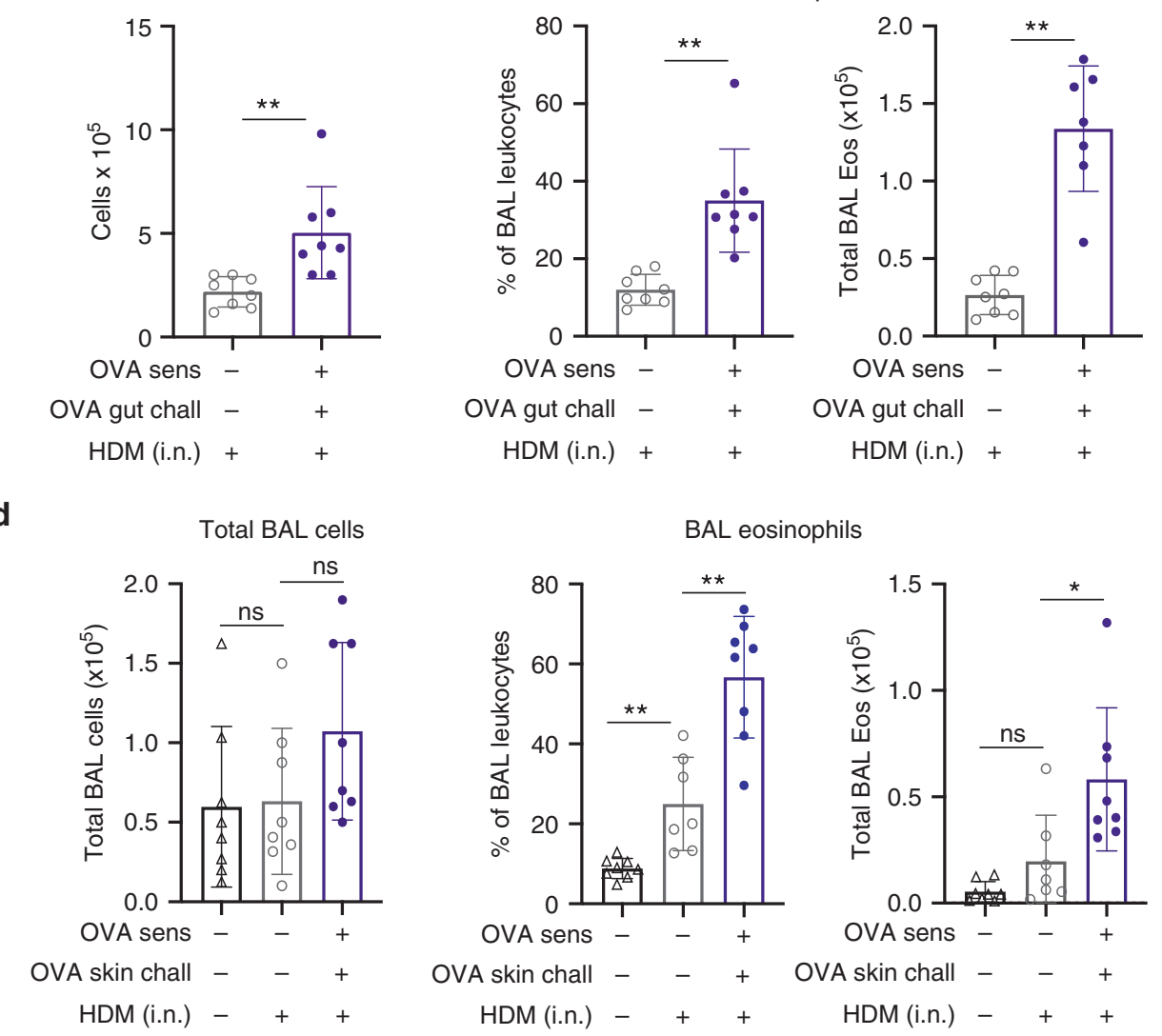

Fig. 6 Remote allergen challenge primes for respiratory allergic disease. a Experimental timeline. OVA- or sham-sensitized mice were challenged with OVA or PBS, respectively, by oral gavage $(\mathbf{b}, \mathbf{c})$ or via skin $(\mathbf{d})$ as described in Fig. 1a. Beginning 4 days after final allergen (or PBS) challenge, mice received six intranasal instillations of house dust mite (HDM) or PBS vehicle control, spaced over 2 weeks as indicated. Mice were sacrificed 1 day following the final HDM instillation. b Representative lung histology sections stained with H\&E. Magnification $\times 200$, scale bar $200 \mu \mathrm{m}$. c, d Assessment of BAL fluid revealed an increase in total BAL cells, and in BAL eosinophils (expressed as a percentage of total BAL leukocytes and as total eosinophils) in mice receiving prior OVA challenge to the gut (c) or skin (d). ${ }^{*} p \leq 0.05$; ${ }^{* *} p \leq 0.005$.

housing conditions with food and water provided ad libitum. Experiments were performed with age- and gender-matched female and male mice.

\section{OVA allergen sensitization}

Mice were sensitized by intraperitoneal injections on days 0,7 , and 14 with 50 ug OVA (Sigma \#A2512) and $0.4 \mathrm{mg}$ alum (Imject Alum, Thermo Scientific \#77161) in $100 \mu \mathrm{L}$ sterile PBS. Control mice were sham-sensitized with alum in PBS. Following sensitization mice were challenged according to one of the methods below.

Allergen challenge methods

Pulmonary allergen challenges were performed using a noninvasive endotracheal spray application originally developed for vaccine delivery systems $\mathrm{s}^{37}$ and modified here for allergen delivery. Mice were anesthetized with ketamine $(50 \mathrm{mg} / \mathrm{mL})$ and xylazine $(5 \mathrm{mg} / \mathrm{mL}$ ) before positioning on a Mouse Intubation Platform. A 
MicroSprayer aerosolizer (model $1 \mathrm{~A}-1 \mathrm{C}$ ) with high pressure syringe (Penn Century, Wyndmoor, PA) was inserted $\sim 1.25 \mathrm{~cm}$ to intubate mice and deliver $50 \mu \mathrm{L}$ of OVA $(20 \mu \mathrm{g})$ in sterile PBS or PBS alone as an air-free aerosol directly into the trachea at approximately the level of the carina (Supplementary Fig. 4a). In some experiments $0.6-6.0 \%$ India ink solutions were substituted for the antigen and lungs harvested for evaluation of the depth and pattern of ink staining $20 \mathrm{~min}$ after inoculation (Supplementary Fig. 4b). To confirm administered aerosol was restricted to lower airways, the oral cavity, esophagus, stomach (including contents), and small bowel were examined and found to be void of India ink (not shown). Of note, it is plausible that self-clearing mechanisms associated with the respiratory mucociliary escalator may, as in humans, deliver a fraction of the airway-deposited allergen to intestinal tissues over time.

Epicutaneous allergen challenge was performed as described. ${ }^{33}$ Briefly, dorsal skin of anesthetized mice was shaved and tapestripped six times with Tegaderm (3 m Healthcare, St. Paul, MN). Overall, $100 \mu \mathrm{g}$ of OVA in $100 \mu \mathrm{L}$ of PBS or PBS alone was applied directly onto tape-stripped skin and covered with a piece of sterile gauze (Covidien, Mansfield, MA) secured to the skin with Tegaderm. Mice were monitored $24 \mathrm{~h}$ after allergen exposure to ensure gauze and Tegaderm strips remained intact, at which point the gauze was removed to avoid mice ingesting allergen-coated gauze during grooming.

Intragastric allergen challenged was achieved by oral gavage (22-gauge needle) with $50 \mathrm{mg}$ of OVA (Sigma-Aldrich, St. Louis, $\mathrm{MO})$ in $250 \mu \mathrm{L}$ of sterile PBS. Parallel experiments with india ink dye confirm dye is confined to the stomach following the oral gavage technique, with no evidence of dye staining along the length of the esophagus (Supplementary Fig. 4c).

Recovery of tissues

At indicated time points mice were euthanized, cardiac blood collected for cell differential counts and lung, small intestine, and skin samples collected into ice-cold PBS. For histological analyses skin samples from the mid-dorsal back, longitudinal sections from the middle of the left lung lobe and rings of small intestinal tissues were fixed in $10 \%$ neutral buffered formalin and embedded in paraffin. Four-micron sections were stained with H\&E for routine analyses or PAS (lung tissues) for analysis of mucus.

Eosinophil enumeration by histology

Eosinophils were identified within lung and intestinal tissues by immunohistochemistry staining after antigen retrieval with an eosinophil specific rat anti-mouse major basic protein monoclonal antibody (MBP, provided by Dr. Beth Jacobsen and the Lee Laboratory, Mayo Clinic, Arizona) as previously described, ${ }^{38}$ followed by counterstaining with methyl green. In some parallel experiments eosinophils were enumerated from slides stained with Fast Green $(0.2 \%$ in $70 \%$ ethanol; Sigma-Aldrich, St. Louis, $\mathrm{MO})$ and Neutral Red (0.5\% in water, Fluka, St. Louis, MO). Preliminary studies confirmed that although MBP immunohistochemistry increased the overall detection of eosinophils across all groups, statistical comparisons between groups were similar regardless of staining method used (Supplementary Fig. 5). For all studies eosinophils were quantified from viewer-blinded slides.

Determining eosinophil frequency by flow cytometry

To determine frequency of eosinophils within whole tissues, lung or small intestine were digested to achieve single-cell suspensions and assessed by flow cytometry. For lung digests, lungs were perfused with $10 \mathrm{~mL}$ of PBS injected through the right ventricle prior to recovery. Minced lung tissues were digested with $175 \mathrm{U} / \mathrm{mL}$ collagenase IV (Worthington Biomedical Corporation), mechanically disrupted and passed through $40 \mu \mathrm{m}$ filters. Residual red blood cells were lysed with BD PharmLyse (BD Biosciences) per the manufacturer's instructions. For intestinal tissue digests, small intestine was harvested as described. ${ }^{12}$ Briefly, intestinal fragments $(\sim 5 \mathrm{~mm})$ were incubated in DTE buffer $(10 \%$ fetal bovine serum, $1 \mathrm{mM}$ HEPES, $2.5 \mathrm{mM} \mathrm{NaHCO}, 1 \mathrm{mM}$ DTT in HBSS-/-) at $37^{\circ} \mathrm{C}$ with shaking to release IE cells. Tissue fragments were then incubated in EDTA-containing buffer ( $1 \mathrm{X}$ penicillin/streptomycin, $1.3 \mathrm{mM}$ EDTA in HBSS-/-) at $37^{\circ} \mathrm{C}$ with shaking to release epithelial cells, which were discarded. Tissue fragments were then washed and incubated in $1 \mathrm{U} / \mathrm{mL}$ collagenase 1 at $37^{\circ} \mathrm{C}$ for $30 \mathrm{~min}$ with shaking. Digested tissue was passed through a $70 \mu \mathrm{m}$ strainer and collected as LP fraction. IE and LP cell pellets were resuspended in $44 \%$ Percoll, underlayed with $67 \%$ Percoll, and centrifuged for $20 \mathrm{~min}$ at $1000 \times \mathrm{g}$. Enriched viable IE or LP cells were collected from the interphase and stained for flow cytometry. LIVE/DEAD Fixable Aqua Dead Cell Stain Kit (Invitrogen, Grand Island, NY), CD45-PE-Cy7 (30-F11), CD4-APC (RM 4-5) and SiglecF-PE (E50-2440) from BD Biosciences (San Jose, CA), and CD11c-FITC or CD11c-APC (N418) and CD8-FITC (53-6.7) from BioLegend (San Diego, CA) were used. Data were acquired using LSR II (BD Biosciences, San Jose, CA) or Gallios flow cytometers and analyzed with FlowJo analysis software (Tree Star, Ashland, OR).

House dust mite-induced allergic airway inflammation

Following OVA sensitization and challenge mice received six intranasal exposures spread over two weeks (see Fig. 6a) to D. pteronyssinus HDM extract (Stallergenes Greer Laboratories, Lenoir, $\mathrm{NC}$ ), each intranasal inoculum containing $3 \mu \mathrm{g}$ of HDM (based on protein weight) in $35 \mu \mathrm{L}$ sterile PBS. Intranasal HDM was administered to anesthetized mice which were held in the supine position for $10 \mathrm{~s}$ following inhalation. Mice were sacrificed 1 day after final HDM administration, and BAL fluid collected into cold PBS containing $2.5 \%$ bovine serum albumin (BSA, Sigma \#A7030).

\section{Statistical analyses}

Data are presented as mean \pm standard deviation for each experimental group. Statistical analyses were performed using one-way analysis of variance or two-tailed Student's $t$ test as indicated.

\section{ACKNOWLEDGEMENTS}

Authors thank Michiko Oyoshi, PhD, Boston Children's Hospital for technical advice in establishing the skin challenge model. This work was supported by NIH R01Al121186 to L.A.S

\section{AUTHOR CONTRIBUTIONS}

L.A.S. conceived the project and supervised the work. C.L.O., M.B-B., J.J.X., and L.A.S. designed experiments. C.L.O., M.B-.B., J.J.X., S.M., E.C., J.F., Q.Y., N.G., R.M., and K.D. performed experiments. C.L.O., M.B.-B., J.J.X., and L.A.S. analyzed data. C.L.O. and L.A.S. wrote the manuscript.

\section{ADDITIONAL INFORMATION}

The online version of this article (https://doi.org/10.1038/s41385-020-0310-x) contains supplementary material, which is available to authorized users.

Competing interests: The authors declare no competing interests.

Publisher's note Springer Nature remains neutral with regard to jurisdictional claims in published maps and institutional affiliations.

\section{REFERENCES}

1. Bantz, S. K., Zhu, Z. \& Zheng, T. The atopic march: progression from atopic dermatitis to allergic rhinitis and asthma. J. Clin. Cell Immunol. 5, 202 (2014).

2. Guilbert, T. W. et al. Atopic characteristics of children with recurrent wheezing at high risk for the development of childhood asthma. J. Allergy Clin. Immunol. 114, 1282-1287 (2004). 
3. Gustafsson, D., Sjoberg, O. \& Foucard, T. Development of allergies and asthma in infants and young children with atopic dermatitis-a prospective follow-up to 7 years of age. Allergy 55, 240-245 (2000).

4. Kapoor, R. et al. The prevalence of atopic triad in children with physicianconfirmed atopic dermatitis. J. Am. Acad. Dermatol. 58, 68-73 (2008).

5. Kulig, M. et al. Natural course of sensitization to food and inhalant allergens during the first 6 years of life. J. Allergy Clin. Immunol. 103, 1173-1179 (1999).

6. Martinez, F. D. et al. Asthma and wheezing in the first six years of life. The Group Health Medical Associates. N. Engl. J. Med. 332, 133-138 (1995).

7. Novembre, E. et al. Natural history of "intrinsic" atopic dermatitis. Allergy 56, 452-453 (2001)

8. Ricci, G. et al. Long-term follow-up of atopic dermatitis: retrospective analysis of related risk factors and association with concomitant allergic diseases. J. Am. Acad. Dermatol. 55, 765-771 (2006).

9. Spergel, J. M. From atopic dermatitis to asthma: the atopic march. Ann. Allergy Asthma Immunol. 105, 99-106 (2010). quiz 7-9, 17.

10. van der Hulst, A. E., Klip, H. \& Brand, P. L. Risk of developing asthma in young children with atopic eczema: a systematic review. J. Allergy Clin. Immunol. 120, 565-569 (2007).

11. Powell, N., Walker, M. M. \& Talley, N. J. Gastrointestinal eosinophils in health, disease and functional disorders. Nat. Rev. Gastroenterol. Hepatol. 7, 146-156 (2010).

12. Xenakis, J. J. et al. Resident intestinal eosinophils constitutively express antigen presentation markers and include two phenotypically distinct subsets of eosinophils. Immunology 154, 298-308 (2018).

13. Carlens, J. et al. Common gamma-chain-dependent signals confer selective survival of eosinophils in the murine small intestine. J. Immunol. 183, 5600-5607 (2009).

14. Mesnil, C. et al. Lung-resident eosinophils represent a distinct regulatory eosinophil subset. J. Clin. Investig. 126, 3279-3295 (2016).

15. Percopo, C. M. et al. SiglecF $+\mathrm{Gr} 1$ hi eosinophils are a distinct subpopulation within the lungs of allergen-challenged mice. J. Leukoc. Biol. 101, 321-328 (2017).

16. Rose, W. A. 2nd, Okragly, A. J., Patel, C. N. \& Benschop, R. J. IL-33 released by alum is responsible for early cytokine production and has adjuvant properties. Sci. Rep. 5, 13146 (2015).

17. Johnston, L. K. \& Bryce, P. J. Understanding interleukin 33 and its roles in eosinophil development. Front. Med. 4, 51 (2017).

18. Marichal, T., Mesnil, C. \& Bureau, F. Homeostatic eosinophils: characteristics and functions. Front. Med. 4, 101 (2017).

19. Abdala Valencia, H., Loffredo, L. F., Misharin, A. V. \& Berdnikovs, S. Phenotypic plasticity and targeting of Siglec-F(high) CD11c(low) eosinophils to the airway in a murine model of asthma. Allergy 71, 267-271 (2016).

20. McBrien, C. N. \& Menzies-Gow, A. The biology of eosinophils and their role in asthma. Front. Med. 4, 93 (2017).

21. Dunican, E. M. et al. Mucus plugs in patients with asthma linked to eosinophilia and airflow obstruction. J. Clin. Investig. 128, 997-1009 (2018).
22. Hoggatt, A. F., Hoggatt, J., Honerlaw, M. \& Pelus, L. M. A spoonful of sugar helps the medicine go down: a novel technique to improve oral gavage in mice. J. Am. Assoc. Lab Anim. Sci. 49, 329-334 (2010).

23. Jung, Y. \& Rothenberg, M. E. Roles and regulation of gastrointestinal eosinophils in immunity and disease. J. Immunol. 193, 999-1005 (2014).

24. Mishra, A., Hogan, S. P., Lee, J. J., Foster, P. S. \& Rothenberg, M. E. Fundamental signals that regulate eosinophil homing to the gastrointestinal tract. J. Clin. Investig. 103, 1719-1727 (1999).

25. Bui, L. K., Hayashi, T., Nakashima, T. \& Horii, Y. Eosinophilic venulitis in the small intestines in a mouse model of late asthma. Inflammation 34, 499-508 (2011).

26. Leyva-Castillo, J. M. et al. Mechanical skin injury promotes food anaphylaxis by driving intestinal mast cell expansion. Immunity 50, 1262-75 e4 (2019).

27. $L i, M$. Current evidence of epidermal barrier dysfunction and thymic stromal lymphopoietin in the atopic march. Eur. Respir. Rev. 23, 292-298 (2014).

28. Benninger, M. S., Strohl, M., Holy, C. E., Hanick, A. L. \& Bryson, P. C. Prevalence of atopic disease in patients with eosinophilic esophagitis. Int. Forum Allergy Rhinol. 7, 757-762 (2017).

29. Jensen, E. T., Martin, C. F., Kappelman, M. D. \& Dellon, E. S. Prevalence of eosinophilic gastritis, gastroenteritis, and colitis: estimates from a national administrative database. J. Pediatr. Gastroenterol. Nutr. 62, 36-42 (2016).

30. Lucendo, A. J., Arias, A. \& Tenias, J. M. Relation between eosinophilic esophagitis and oral immunotherapy for food allergy: a systematic review with meta-analysis. Ann. Allergy Asthma Immunol. 113, 624-629 (2014).

31. Semancik, E. \& Sayej, W. N. Oral immunotherapy for peanut allergy induces eosinophilic esophagitis: three pediatric case reports. Pediatr. Allergy Immunol. 27, 539-541 (2016)

32. Akei, H. S., Mishra, A., Blanchard, C. \& Rothenberg, M. E. Epicutaneous antigen exposure primes for experimental eosinophilic esophagitis in mice. Gastroenterology 129, 985-994 (2005).

33. Venturelli, N. et al. Allergic skin sensitization promotes eosinophilic esophagitis through the IL-33-basophil axis in mice. J. Allergy Clin. Immunol. 138, 1367-80 e5 (2016).

34. Liu, A. H. et al. National prevalence and risk factors for food allergy and relationship to asthma: results from the National Health and Nutrition Examination Survey 2005-2006. J. Allergy Clin. Immunol. 126, 798-806 e13 (2010).

35. Sampson, H. A. Update on food allergy. J. Allergy Clin. Immunol. 113, 805-819 (2004). quiz 20

36. James, J. M. Respiratory manifestations of food allergy. Pediatrics $\mathbf{1 1 1}(6 \mathrm{Pt} \mathrm{3}$ ), 1625-1630 (2003).

37. Bivas-Benita, M., Zwier, R., Junginger, H. E. \& Borchard, G. Non-invasive pulmonary aerosol delivery in mice by the endotracheal route. Eur. J. Pharm. Biopharm. 61 214-218 (2005).

38. Ochkur, S. I. et al. Coexpression of IL-5 and eotaxin-2 in mice creates an eosinophil-dependent model of respiratory inflammation with characteristics of severe asthma. J. Immunol. 178, 7879-7889 (2007). 